
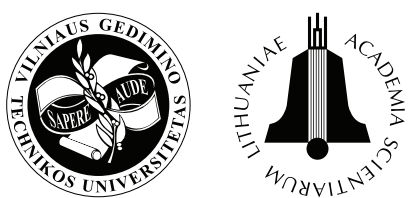

TRANSPORT

2010

25(1): 29-35

\title{
FINDING A SOLUTION FOR A COMPLEX STREET ROUTING PROBLEM USING THE MIXED TRANSPORTATION MODE
}

\author{
Peter Matis \\ Dept of Transportation Networks, Faculty of Management Science and Informatics, \\ University of Zilina, Univerzitna 8215/1, 01026 Zilina, Slovak Republic \\ E-mail: Peter.Matis@fria.utc.sk
}

Received 6 June 2009; accepted 1 February 2010

\begin{abstract}
The Street Routing Problem (SRP) is a special case of the well-known Vehicle Routing Problem (VRP). The goal is to service a large number of customers in the city zone. New heuristics for solving a complex SRP is evaluated based on real data. This paper presents several approximations to the length of SRP using the mixed transportation mode and compares them with the published approximations used for VRP or Travelling Salesman Problems (TSP). The system was tested in five real world instances ranging from 12000 to 29000 customers.
\end{abstract}

Keywords: street routing problem (SRP), vehicle routing problem (VRP), heuristics, approximations.

\section{Introduction}

Postal delivery in agglomerations with large numbers of customers is one of the most difficult operational problems faced by local delivery companies. This paper describes a Decision Support System (DSS) for postal delivery in towns with more than 100000 inhabitants with the object of designing service districts and vehicle collection routes subject to a number of operational constraints. The reported results are abstracted and do not consider several very special issues.

The problem of postal delivery optimization can be modelled by Street Routing Problems (SRP) and Capacitated Vehicle Routing Problems (CVRP) (Matis 2007 and 2008; Janáček 2003; Janáček and Gábrišová 2009; Antov et al. 2009; Çalışkanelli et al. 2009; Jovanović et al. 2009; Junevičius and Bogdevičius 2007, 2009; Szücs 2009; Turskis et al. 2009; Baublys 2008; Brauers et al. 2008; Daunoras et al. 2008; Gowri and Sivanandan 2008; Niewczas et al. 2008; Tanczos and Torok 2007; Ziari et al. 2007; Poot et al. 2002).

A typical SRP has thousands of customers. I want to find and evaluate solutions for cases with more than 10000 customers. In such large agglomerations, service is performed using a mixed transportation mode. Service personnel are driven by car to the first point of the serviced area, serve the area on foot and then returns to the depot by car. To find a good feasible solution for these cases, I identified two stages of the problem. First, I need to aggregate customers to natural clusters and find an approximate length of SRP for these clusters.
Second, I need to solve a special case of CVRP of finding good car routes. The problem is defined over the street network of the region.

SRP systems could be solved by integrating heuristics with a visual and database environment such as Geographical Information Systems (GIS) (for example, Burinskienė 2009; Jakimavičius and Burinskienè 2009; Mesarec and Lep 2009). in SRP.

This paper discusses a GIS-based DSS for routing

SRP is a less explored group of problems than Vehicle Routing Problems (VRP) and there is not a good, publicly available sample of data for method comparison. The authors, for example (Ruiz et al. 2004), usually solve specific problems and it is difficult to compare solutions across these problems as each of them is somewhat unique.

Therefore, I find it necessary to use my own experimental sample, one that covers most of typical SRP and at the same time, makes it possible to compare each of the solution methods. My experimental sample was taken from five large cities and surrounding villages in Slovakia. The customers were houses in these cities and full street infrastructure is available. The cities included Bratislava, Kosice, Zilina, Presov and Nitra. The number of customers in these four agglomerations varies from 12000 to 29000 .

Data was collected manually from source maps ZM 1:10 000 purchased from the Geodetic and Cartographic Institute of the Slovak Republic. A part of data was col- 
lected using GPS receivers and is not publicly available at the moment.

The remainder of this paper is arranged as follows. First, a definition of the problem is given. Then, new aggregation methods are presented and a method for the approximation of route length for SRP is introduced and compared to the existing methods. Section four presents a possible solution for a special case of CVRP for sending postmen to districts. Section five presents the use of DSS and GIS to work towards practical solutions for the stated problem. Conclusions are drawn in section six.

\section{Definition of Complex SRP Using the Mixed Transportation Mode}

In recent years, we have seen a number of centralization activities in postal delivery companies to improve the performance of their processes. This creates a large number of customers served from one central depot. The centralization of delivery operations in one depot for a large number of customers has some advantages and disadvantages. One of the key advantages to centralization is the ability to use more advanced and expensive technologies for preparation before the deliveries are picked up. The preparation of deliveries usually takes more than $10 \%$ of the total working time of postmen. By shortening this time combined with efficient transportation methods for placing postmen in their districts, one can allow more time for delivery and/or reduce the number of postmen.

Fig. 1 presents possible service areas around the city of Presov and its 15797 customers. The area includes Presov and 11 surrounding villages.

The goal is to find good solutions for SRP in such a large and centralized region. For each customer, there is an average daily service time. Postmen can be driven by car to the starting point of their service route and driven back to the depot at the end of the working day. Deliveries are made on foot, so the problem represents a special case of SRP using the mixed transportation mode. Car driver is also a postman and serves one district. There are either no transportation related expenses or they are relatively low compared to other expenses. Delivery time is limited for each postman. Time gaps between the physical end of deliveries and the arrival of the car for returning the postman back to the depot should be tight.

\section{The Aggregation of Customers to Natural Clusters and Approximation of Route Length}

One of the key problems of solving a serious SRP using the mixed transportation mode is to aggregate customers into natural clusters. Good examples of measuring cluster visual attractiveness can be found in Poot et al. (2002) and Matis (2008). Each cluster is then treated as one district for SRP and is served by one postman. The size of the cluster is determined by the estimated length of the SRP route, the travel speed of the postman, delivery time for customers in the cluster and the total time available for serving the cluster.

Although plenty of work seems to be done in the field of aggregation in general, there is not a specific developed method for SRP aggregation. For example, Oppen and Løkketangen (2006) describes the aggregation of variables (columns) in linear problems and the way it can be used for estimating both an optimal solution and an optimal objective value of the original problem. Another example illustrates how Toth and Vigo (2002) uses structural information about city locations to aggregate Travelling Salesman Problems (TSP). By aggregating

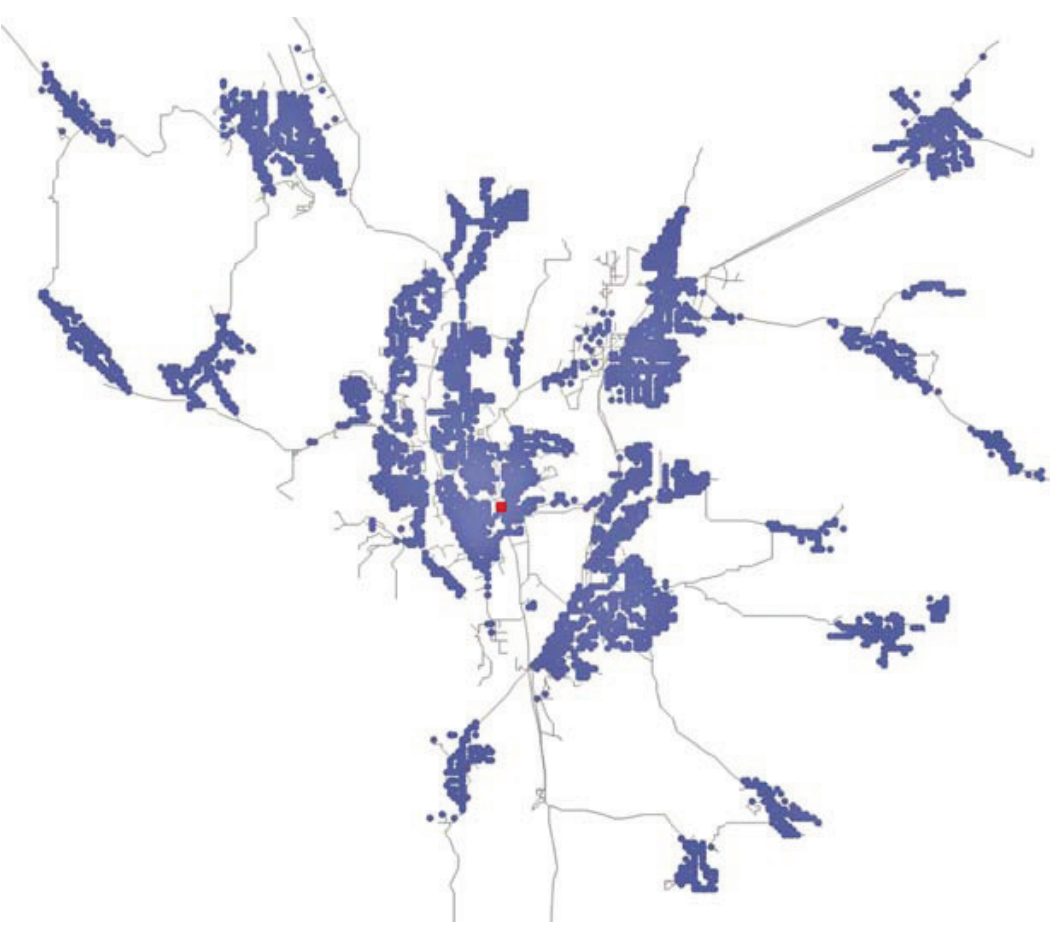

Fig. 1. Customers and a depot in large Presov region 
customers, the size of the problem decreases. On the other hand, one must assume that errors are introduced as customers that do not belong to the same cluster in the optimal solution may be aggregated. The goal will then be to perform aggregation in a way that minimizes these errors but at the same time, sufficiently reduces the size of the problem to allow for better solutions within the same solution time. We have to remember that the two-phase methods usually do not make the use of the sophisticated search algorithms in their procedures. In reality, optimality is not the goal but getting a good solution in a short amount of time is crucial. If aggregation can help in getting such an effective but not necessarily optimal solution quickly, it will be of interest. Facing another problem tackled by Beasley and Christofides (1997) the large mail order company used an approach of aggregating customers into postal districts according to the alphanumeric postal code system used in the United Kingdom. Nevertheless, we cannot apply this method because we are actually re-shaping the postal system for a new postal code system.

The aggregation of customers on a daily basis is a daunting task simply because there are too many customers and their demands change from day to day. Therefore, assignments made in the previous day might not be applicable for the following day. In order to tackle this problem, I create permanent districts. Logic is that a postman then can know his/her district well and reduce possible errors in delivery while simultaneously shortening delivery time.

There is another problem related to aggregation. One logical city unit (vicinity village or city district) can be divided to several service districts and the last one needs only to take a small amount of postman's working time. It is hard to merge these residuum districts to one district because they can be distributed across the whole studied area.

For aggregating customers into natural clusters, I used a part of Fuzzy Cluster Heuristics (FCH) (Matis 2008) that can be implemented considering the following steps:

1. Estimate the minimum number of clusters $p$ needed for serving all customers using the following formula:

$$
p=\frac{\sum_{c=1}^{N} C S T_{c}+\frac{\sum_{s=1}^{M} D_{s}}{V}}{S W T},
$$

where: $\operatorname{CST}_{c}$ is an average service time for customer $c$; $N$ is the number of customers; $M$ is the number of street segments where customers are located; $D_{s}$ is the length of street segment $s ; V$ is an average speed of the walking postman; $S W T$ is the available service time of one postman.

2. Locate $p$ medians, so they are uniformly distributed in the serviced area.

3. Create $p$ clusters of customers around these medians using clustering by 'fuzzy c-means' (FCM).
The membership of customers in each cluster is set as a triangle fuzzy number based on the route distance between the cluster median and the customer.

4. For each cluster, approximate the route length and estimate the average service time.

5. If there are many clusters (more than 20\%) having their service time over the SWT, increase the number of clusters $p$ and go to step 2, otherwise end the algorithm with the resulting clusters and these are used as SRP districts.

In this algorithm, I used an approximation of length for the SRP route of one postman. In our case, this is actually the length of TSP or special TSP where the postman does not necessarily need to return back to the point where his/her route started.

There are some good samples in literature how other authors estimate the length of TSP or VRP (Figliozzi 2008). In my case, I present two estimations from Toth and Vigo (2002):

$$
\begin{aligned}
& \operatorname{TSP}(D)=0.765 \cdot \sqrt{N \cdot A} \\
& \operatorname{TSP}(D)=\left(0.83-0.0011 \cdot(N+1)+\frac{1.11 \cdot S}{N+1}\right) \cdot \sqrt{N \cdot A},
\end{aligned}
$$

where: $\operatorname{TSP}(D)$ is the estimated length of the route, $A$ is an area of the serviced cluster, $S$ is a proportion of the length-width ratio for the outside rectangle of the cluster created in such a way that the ratio is always larger than or equal to 1 .

The authors always use the Euclidean or Manhattan travel metric.

In our case, neither Euclidian nor Manhattan metrics interpret the real distance for the travelling salesman. Our case is specific because the authors focus on VRP and our problem is SRP that has a couple of differences in comparison with VRP (Matis 2006) and some of these differences are related to the distribution of customers and network density. In my model, I used the following estimation of the length for one postman's path:

$$
\operatorname{TSP}(D)=1.445 \cdot S D,
$$

where: $S D$ is a total distance of all street segments served by one postman.

This formula (4) was created from a simple regression using sample data with 78 service clusters compared to the best results from heuristics described by Matis (2008). Fig. 2 and Table 1 show the differences of the estimated length in the best found length ratio for different SRP cases using these three formulas.

In this experiment, I calculated the route length using the TABU search metaheuristic (Matis 2008). The first approximation (see Equation 2) by Toth and Vigo (2002) overestimated length in all cases tested by the TABU search (Matis 2007). The average overestimation was more than $90 \%$ of the total length. The second approximation (see Equation 3) by Toth and Vigo (2002) usually underestimated the total length in all our cases. 


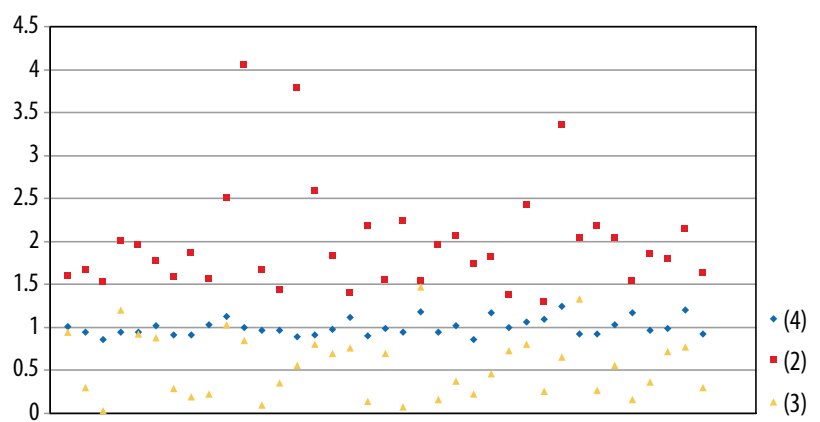

Fig. 2. The ratios of the estimated TSP length for the best found TSP length of different approximations

Table 1. The average ratio of the estimated TSP length for the best found TSP length of different approximations

\begin{tabular}{ccc}
\hline Formula & $\begin{array}{c}\text { Average ratio for estimated length / } \\
\text { The best found TSP length }\end{array}$ & $\begin{array}{c}\text { Standard } \\
\text { deviation }\end{array}$ \\
\hline$(2)$ & 1.99 & 0.615996 \\
\hline$(3)$ & 0.56 & 0.370789 \\
\hline$(4)$ & 1.01 & 0.099281 \\
\hline
\end{tabular}

Due to the fact that on average the ratio was 0.56 , I can assume it was much lower than the optimal route length in most cases. In most cases, the new approximation (see Equation 4) showed a good estimation of the total length as it was demonstrated by low standard deviation against the best found solution. For a comparison, I used the best found solution. The optimal solution is hard to get for most districts in SRP because the districts usually represent several hundred customers. Fig. 3 shows the resulting clusters created by FCH in city of Zilina.

\section{Transportation of Postmen to Districts}

To get a solution for a complex SRP, we need to distribute postmen to their service districts using cars. This is a variation of the Capacitated Vehicle Routing Problem (CVRP) with the addition of extra constraints. One extra constraint is that we have to maximize the utilization of vehicles. An additional constraint is that the vehicle has to return to the depot using the same route. In our cases, all vehicles have the same capacity. The goal is to minimize the total length of routes for all cars and second, to minimize the number of cars necessary for all service districts that are farther from the service centre. Car driver serves the last district and then collects all postmen that were in the car when they started at the service centre. The postman that first leaves the car has more time for delivery than the last one. Thus, I could change my algorithm for creating natural clusters and make larger clusters that are closer to the service centre making further clusters smaller. In presented case, I did not make this change. Difference in time they leave the car is usually less than 10 minutes and because delivery time has a probabilistic character, this may not have an effect on the final result.

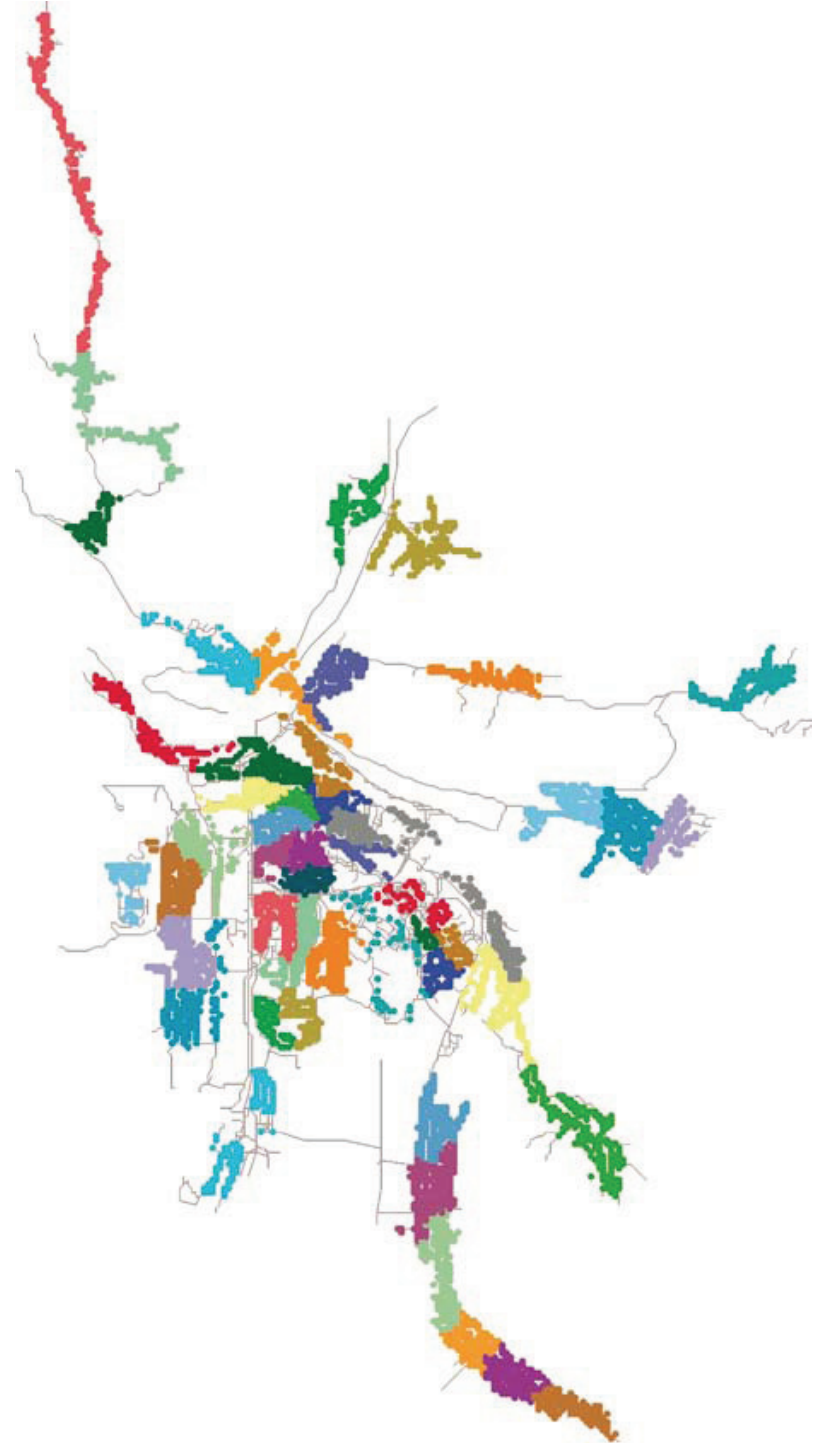

Fig. 3. Natural clusters in large Zilina region created by FCH

There is not a method that could find the optimal solution for this problem for a practical-size SRP. I used the TABU search (TABU) metaheuristic (Matis 2008) that was changed to this specific case where my goal was not only to find the minimum total length but also to maximize the utilization of cars. The heuristic was named the TABU for Postman Collection heuristic (TABUPC).

The major difference between the original TABU and TABUPC is that a number of the serviced clusters in the route are limited by a maximum number of passengers in the car. For calculating the length of one trip, we only use the lengths of segments from the depot until the last customer. The car has to return back to the depot using the same route.

The algorithm is based on the ideas put forward by Crainic and Gendreau (2002). The initial solution is generated by simple best position heuristics. Every customer is inserted into the first tour that has enough capacity left over. The customer is inserted into the tour in the best possible way, i.e. so that an increase in distance is 
minimized. If no tour with enough free capacity can be found, a new tour is created which gives a starting solution where all the tours are feasible.

Moves are performed by applying an operator that removes a customer from one route and inserts him/her into another tour. When a customer is removed from a tour, the tour is reconnected by linking both the predecessor and the successor of the removed customer. Let $A(s)=\{(i, v)$ : customer $i$ is visited by vehicle $v\}$ be an attribute set associated with each solution $s$. The insertion of a customer into a tour is done so as to minimize an increase in the length of the tour without changing the order of the customers already available in the tour. The move is evaluated according to the evaluation function:

$$
e(s)=D(s)+\mu \cdot Q(s)+\lambda \cdot \bar{Q}(s),
$$

where $e(s)$ is a value of the evaluation function for solution $s$ after the move; $D(s)$ is the total distance of all tours after the move; $\mu, \lambda$ are positive parameters; $\mu$ is dynamically adjusted during search; $Q(s)$ is the total violation of capacity constraints for tours; $\bar{Q}(s)$ is the total capacity not used for cars but for tours.

To diversify search, any solution that has a higher value of $e(s)$ compared to the solution before the move is given penalty $p(s)=\omega \cdot \delta_{i k}$ that is added to $e(s)$. Here, $\delta_{i k}$ is the number of times when attribute $(i, k)$ has been a part of a good solution, i.e. a solution that is feasible and has a total length less than $\eta$ times of the length of the best solution found so far. Parameter $\omega$ is used for controlling the intensity of diversification. These penalties are used for leading search into less explored parts of solution space whenever a local optimum is found.

TABU search starts from the initial solution, moves at each iteration to the solution that is non-tabu and minimizes $e(s)$. At each step, attribute $(i, k)$ that was removed from $A(s)$ is now declared tabu for several iterations (in our case, 20). During these iterations, it is not allowed to move customer $i$ back to tour $k$. By using a simple aspiration criterion, a tabu move can still be chosen if this leads to a solution that is the best found thus far in the search and is feasible. After each move, the values of parameters $\mu$ and $\lambda$ are adjusted. If the current solution is feasible, the value of $\mu$ is decreased to make it less costly to visit an infeasible solution. In the opposite case, $\mu$ is increased to lead the search back into the feasible region of the solution space. If the current solution is feasible and has a total length less than $\eta$ times of the length of the best feasible solution found so far during the search and the number of iterations performed has reached 200, the solution is considered good. Whenever a good solution is found, the values for $\delta_{i k}$ attributes of the found solution are incremented. The search continues until a defined number of moves have been performed. Fig. 4 shows the centers of clusters created by FCH in city of Presov and routes for delivery cars.

\section{Use of GIS as a Decision Support System for SRP}

Recent developments in street routing problems show that there is a need to make routing software a part of the larger system. One of the possible solutions is to integrate routing software within GIS that can be helpful

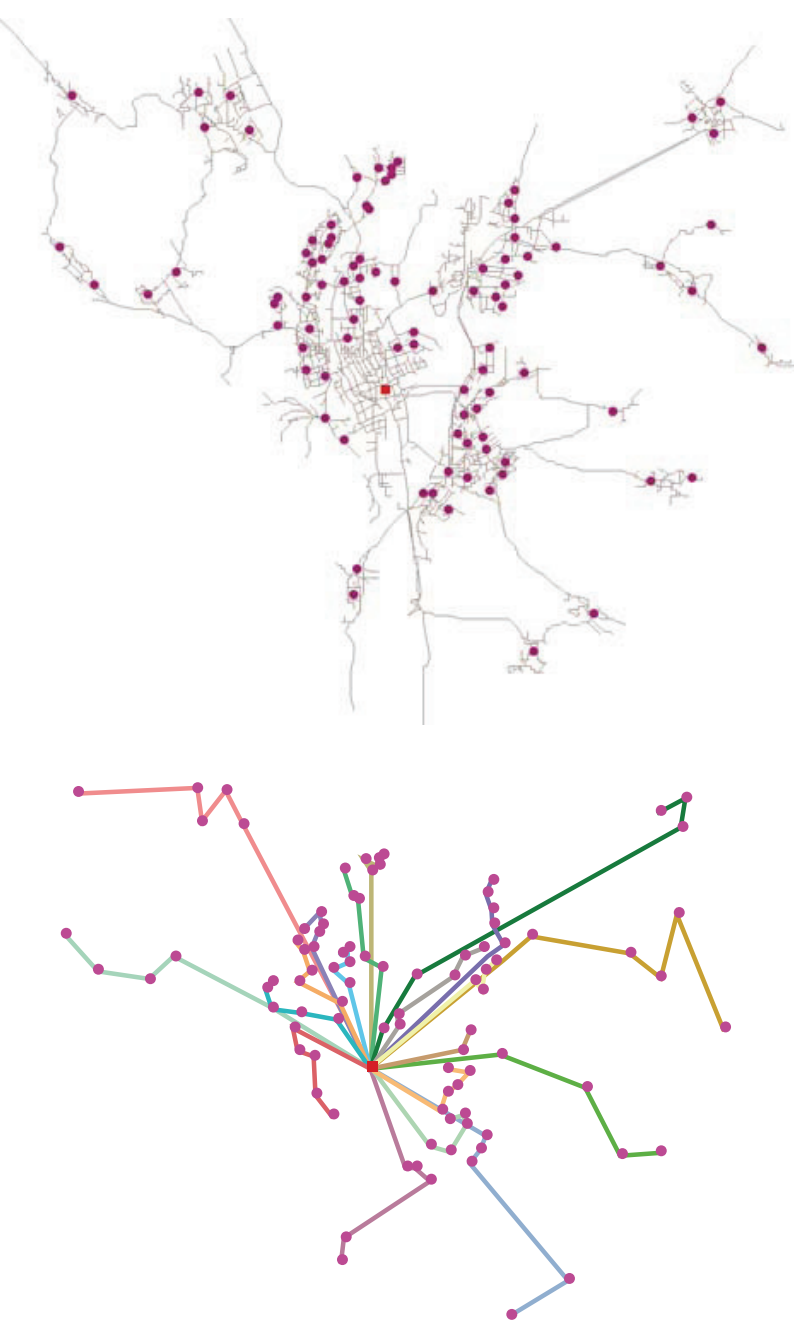

Fig. 4. 94 centres for natural clusters in large Presov region served by cars and routes for twenty cars

in the collection, storing and management of large geographical databases used in routing software. GIS can also be used for creating all outputs from routing software. One of the key properties of GIS for using routing software is its interactive and user-friendly environment. Routing software can find a good solution and explore possibilities while an expert can change the calculated routes to explore other possibilities based on the expert's judgment.

GIS has several useful features that could help with improving the performance of routing software only a few of which can be mentioned here and include safe database management, flexible symbols, map management, drawing capabilities, safety and interoperability.

Decisions support systems (DSS) are important for solving real SRP. Software can be used for working out a solution for the problem as well as for exploring several other possibilities or seeing how changes in the transportation network, regulations or policies could affect routes, expenses and other parameters. Currently, two trends in the development of DSS for SRP exist:

- Independent software packages specialized for SRP with the limited amount of DSS capabilities. Here we can note software like GeoRoute 
produced by the Canadian software company GIRO and the American TRANSCAD having some similarities to GeoRoute that is more feasible for solving SRP because it is specialized for street routing. TRANSCAD is more specialized in node routing.

- The integrated systems based on GIS or CAD. As an example, we can cite ArcView with its ArcGIS Network Analyst. It specializes in node routing. The user can implement extension and then make it an integral part of the whole system.

To shorten development time, I have chosen a standard GIS environment as a base system for data management. The integrator represents the main user interface controlling each heuristic - the connection of GIS database to/from heuristics. In some cases, heuristics could be replaced by regular solvers, for example XPRESS. I used ArcView by ESRI as the main GIS tool because it is an open system and allows the user to program its functionalities. GIS is managed from the integrator by the Avenue scripting language, C\# and VBasic depending on the version of ArcView. GIS is used in the system as a data management tool, editor and for the visualization of results.

\section{Conclusions}

There are expanding activities that can be presented as street-based tasks in the cities. I introduced the methodology of solving specific SRP with a very large number of customers. I used samples from five cities for the verification of my methodology and algorithms.

I presented a new heuristic for creating natural clusters and estimating the length of the route. I have not compared the results of heuristics with any other results because there is no publication dealing with this problem. From a small sample, I can conclude that an average calculation time for this part of the solution is $232 \mathrm{sec}$ using a PC with Dual Core Processor Intel Pentium. For most of practical applications, this is acceptable and results are promising.

I created a variation of TABU search heuristic for distributing postmen to their service districts using cars. The obtained results show that heuristic can also be used for the cases with a heterogeneous vehicle park.

I created DSS for solving this specific SRP tested on the sample data only.

Paths for future work to find better ways to solve a complex SRP might be:

- investigation into more sophisticated methods for aggregation;

- using a better estimation of TSP length;

- improving diversification mechanisms for the tabu search algorithm.

\section{Acknowledgement}

This work was supported by the project 'Centre of excellence of information sciences and knowledge systems' ITMS 26220120007

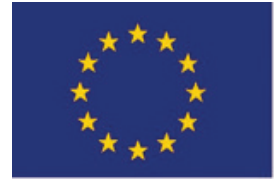

Project Part-Financed by the European Union

European Regional

Development Fund

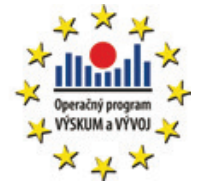

\section{References}

Antov, D.; Abel, K.; Sürje, P.; Rõuk, H.; Rõivas, T. 2009. Speed reduction effects of urban roundabouts, The Baltic Journal of Road and Bridge Engineering 4(1): 22-26. doi:10.3846/1822-427X.2009.4.22-26

Baublys, A. 2008. Model for distribution of warehouses in the commercial network in optimising transportation of goods, Transport 23(1): 5-9. doi:10.3846/1648-4142.2008.23.5-9

Beasley, J. E.; Christofides, N. 1997. Vehicle routing with a sparse feasibility graph, European Journal of Operational Research 98(3): 499-511. doi:10.1016/S0377-2217(96)00048-3

Brauers, W. K. M.; Zavadskas, E. K.; Peldschus, F.; Turskis, Z. 2008. Multi-objective decision-making for road design, Transport 23(3): 183-193.

doi:10.3846/1648-4142.2008.23.183-193

Burinskienè, M. 2009. New methodology for sustainable development towards sustainable transportation system, Technological and Economic Development of Economy 15(1): 5-9. doi:10.3846/1392-8619.2009.15.5-9

Çalışkanelli, P.; Özuysal, M.; Tanyel, S.; Yayla, N. 2009. Comparison of different capacity models for traffic circles, Transport 24(4): 257-264. doi:10.3846/1648-4142.2009.24.257-264

Daunoras, J.; Bagdonas, V.; Gargasas, V. 2008. City transport monitoring and routes optimal management system, Transport 23(2): 144-149. doi:10.3846/1648-4142.2008.23.144-149

Figliozzi, M. A. 2008. Planning approximations to the average length of vehicle routing problems with varying customer demands and routing constraints, Transportation Research Record: Journal of the Transportation Research Board 2089: 1-8. doi:10.3141/2089-01

Crainic, T. G.; Gendreau, M. 2002. Cooperative parallel TABU search for capacitated network design, Journal of Heuristics 8(6): 601-627

Gowri, A.; Sivanandan, R. 2008. Evaluation of left turn channelization at a signalized intersection under heterogeneous traffic conditions, Transport 23(3): 221-229.

doi:10.3846/1648-4142.2008.23.221-229

Jakimavičius, M.; Burinskienè, M. 2009. A GIS and multi-criteria-based analysis and ranking of transportation zones of Vilnius city, Technological and Economic Development of Economy 15(1): 39-48. doi:10.3846/1392-8619.2009.15.39-48

Janáček, J. 2003. Optimalizace na dopravních sitích [Optimization in transport network]. Zilina: Edis. 248 p.

Janáček, J.; Gábrišová, L. 2009. A two-phase method for the capacitated facility problem of compact customer sub-sets, Transport 24(4): 274-282. doi:10.3846/1648-4142.2009.24.274-282

Jovanović, V. D.; Tica, S.; Milovanović, B.; Živanović, P. 2009. Researching and analyzing the features of oil and demand for transporting oil derivates in the area of Belgrade, Transport 24(3): 249-256. doi:10.3846/1648-4142.2009.24.249-256

Junevičius, R.; Bogdevičius, M. 2009. Mathematical modelling of network traffic flow, Transport 24(4): 333-338. doi:10.3846/1648-4142.2009.24.333-338

Junevičius, R.; Bogdevičius, M. 2007. Determination of traffic flow parameters in different traffic flow interaction cases, Transport 22(3): 236-239. 
Matis, P. 2006. Management of street routing problems using decisions support system, Communications 8(3): 5-8.

Matis, P. 2007. The relationship between quantitative and qualitative measurements in solving of street routing problems, in 15th International Scientific Conference on Mathematical Methods in Economics and Industry, Herlany 2007, 144152.

Matis, P. 2008. Decision support system for solving the street routing problem, Transport 23(3): 230-235. doi:10.3846/1648-4142.2008.23.230-235

Mesarec, B.; Lep, M. 2009. Combining the grid-based spatial planning and network-based transport planning, Technological and Economic Development of Economy 15(1): 6077. doi:10.3846/1392-8619.2009.15.60-77

Niewczas, A.; Koszalka, G.; Wrona, J.; Pieniak, D. 2008. Chosen aspects of municipal transport operation on the example of the city of Lublin, Transport 23(1): 88-90. doi:10.3846/1648-4142.2008.23.88-90

Oppen, J.; Løkketangen, A. 2006. Arc routing in a node routing environment, Computers \& Operations Research 33(4): 1033-1055. doi:10.1016/j.cor.2004.09.004

Poot, A.; Kant, G.; Wagelmans, A. P. M. 2002. A Saving based method for real-life vehicle routing problems, Journal of the Operational Research Society 53(1): 57-68.

doi:10.1057/palgrave.jors.2601252

Ruiz, R.; Maroto, C.; Alcaraz, J. 2004. A decision support system for a real vehicle routing problem, European Journal of Operational Research 153(3): 593-606. doi:10.1016/S0377-2217(03)00265-0

Szücs, G. 2009. Developing co-operative transport system and route planning, Transport 24(1): 21-25. doi:10.3846/1648-4142.2009.24.21-25

Tanczos, K.; Torok, A. 2007. Linear optimization model of urban areas' operating efficiency, Transport 22(3): 225-228.

Toth, P.; Vigo, D. 2002. The Vehicle Routing Problem. Philadelphia: Society for Industrial and Applied Mathematics. 367 p.

Turskis, Z.; Zavadskas, E. K.; Peldschus, F. 2009. Multi-criteria optimization system for decision making in construction design and management, Inzinerine Ekonomika - Engineering Economics 1(61): 7-17.

Ziari, H.; Keymanesh, M. R.; Khabiri, M. M. 2007. Locating stations of public transportation vehicles for improving transit accessibility, Transport 22(2): 99-104. 